\title{
Impact of Shock-Induced Lipid Nanobubble Collapse on a Phospholipid Membrane
}

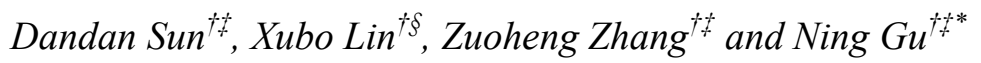

$†$ State Key Laboratory of Bioelectronics and Jiangsu Key Laboratory for Biomaterials and

Devices, School of Biological Science \& Medical Engineering, Southeast University, Nanjing 210096, China

\$Collaborative Innovation Center of Suzhou Nano-Science and Technology, Suzhou Key Laboratory of Biomaterials and Technologies, Suzhou 215123, China

$\S$ Department of Integrative Biology \& Pharmacology, Medical School, The University of Texas Health Science Center at Houston, Texas 77030, USA

* Corresponding author.

Fax: +86(0)25-8327-2460. E-mail: guning@seu.edu.cn. 


\section{The averaged nanojet velocity}

Both Ohl et al. and Vedadi et al. used a equation, $v_{j e t}=l_{j e t} / t_{j e t}$, $\left(l_{j e t}\right.$ was jet length and $t_{j e t}$ was persistence time), to describe a lower bound of the nanojet velocity and inferred that the maximum velocity would be greater. ${ }^{1,2}$ The nanojet velocity was estimated from the fact that the water particles in contact with the proximal side of the bubble had to move into the tip of the nanojet. In our lipid nanobubble systems, we derived the averaged nanojet velocity by dividing the translocation distance of water particles by the translocation time, $v_{j e t}=l_{p} / t_{p}$, where $l_{p}$ was the displacement of water particles initially adjacent to the proximal edge of the bubble during the time of $t_{p}$. Figure $\mathbf{S 3}$ shows the averaged velocities of the nanojets in our lipid nanobubble systems. The nanojet velocity increased as the initial nanobubble diameter increased.

\section{References:}

(1) Ohl, C. D.; Ikink, R. Shock-wave-induced jetting of micron-size bubbles. Phys. Rev. Lett. 2003, 90, 214502.

(2) Vedadi, M.; Choubey, A.; Nomura, K.; Kalia, R. K.; Nakano, A.; Vashishta, P. Structure and dynamics of shock-induced nanobubble collapse in water. Phys. Rev. Lett. 2010, 105, 014503. 


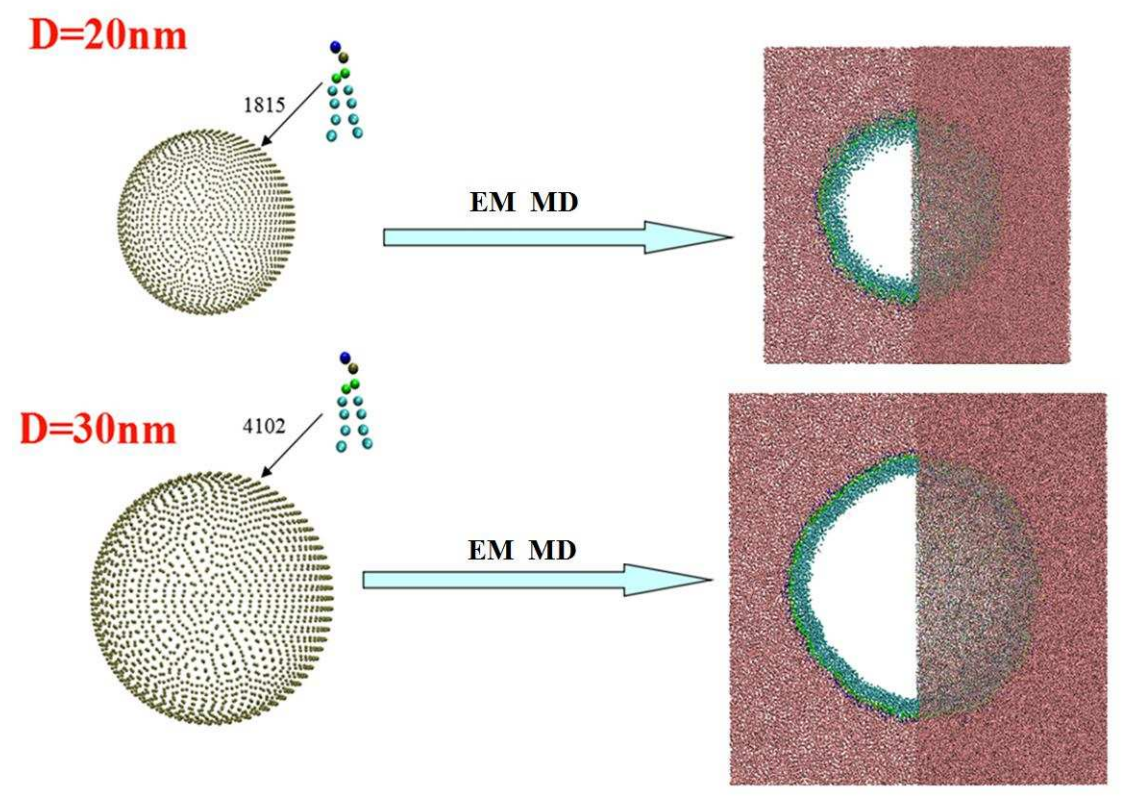

Figure S1. Preparation of lipid nanobubbles. Lipid nanobubbles with diameters $20 \mathrm{~nm}$ or $30 \mathrm{~nm}$ were created by aligning lipid molecules in homogeneous spherical configurations. The processes were realized by a $\mathrm{C}++$ program. After solvation, energy minimization and equilibration simulations, two sizes of stable lipid nanobubbles were achieved. The left sides in the lipid nanobubble systems are cross section, clearly presenting the monolayer structures of the lipid nanobubbles. 


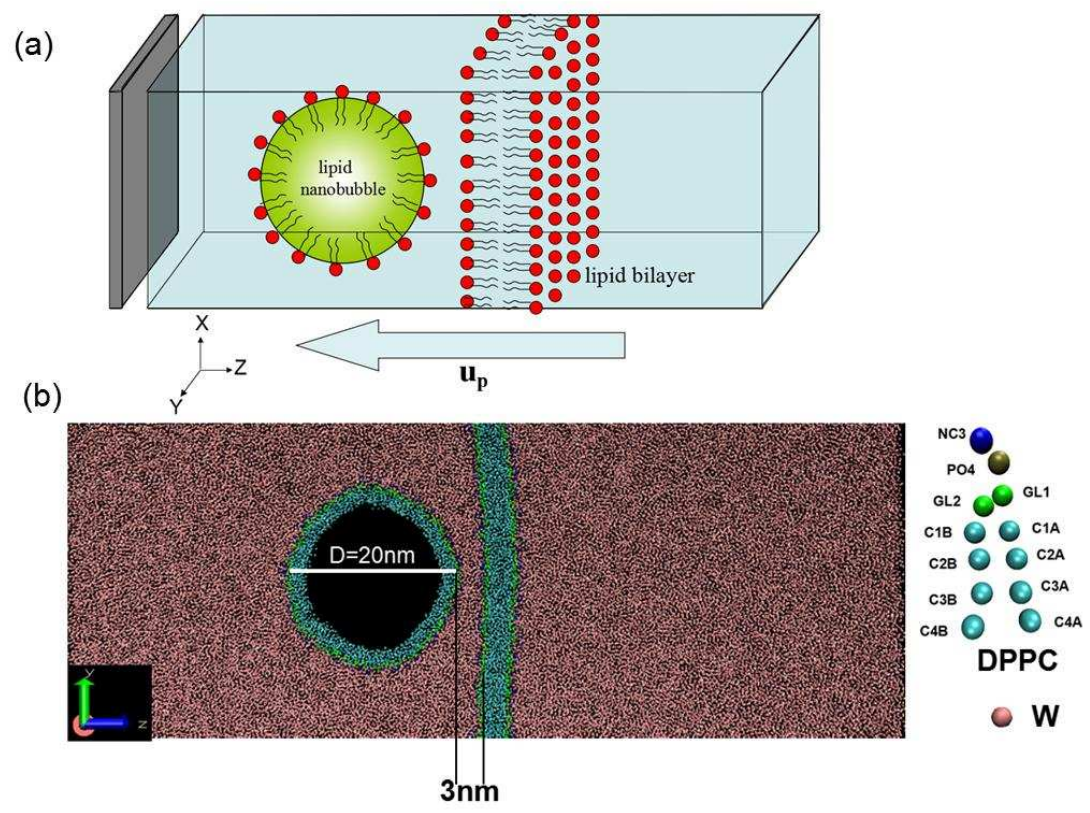

Figure S2. Schematic of a simulation box. A lipid nanobubble was set near the lipid bilayer.

The grey plate was the momentum mirror. The distance of the nanobubble from the lipid bilayer is defined as the minimum distance between the nanobubble edge and the bilayer surface. 


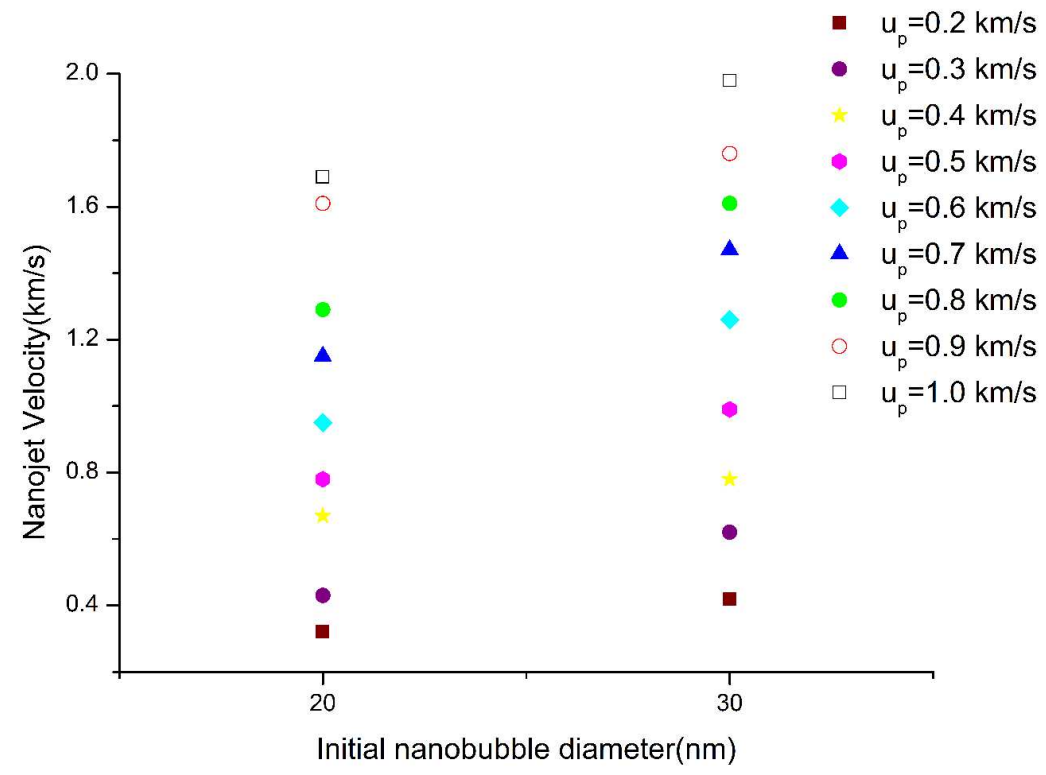

Figure S3. Averaged velocities of the nanojets versus the initial nanobubble diameters.

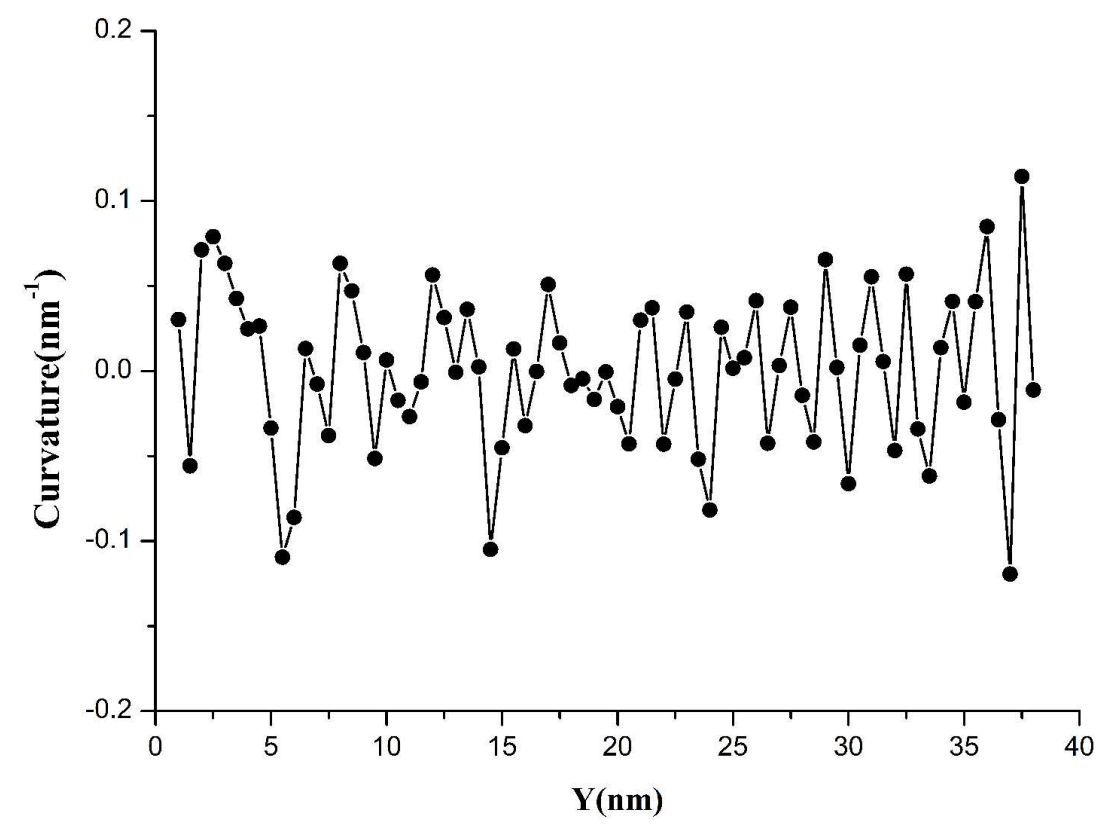

Figure S4. Local curvature of the bilayer for the system with $u_{p}=1.0 \mathrm{~km} / \mathrm{s}$ and D $=30 \mathrm{~nm}$. 